\title{
Identification and Organization of Task Complexity Factors Based on a Model Combining Task Design Aspects and Complexity Dimensions
}

\author{
Dong-Han Ham \\ Department of Industrial Engineering, Chonnam National University, Gwangju, 500-757
}

\begin{abstract}
Objective: The purpose of this paper is to introduce a task complexity model combining task design aspects and complexity dimensions and to explain an approach to identifying and organizing task complexity factors based on the model. Background: Task complexity is a critical concept in describing and predicting human performance in complex systems such as nuclear power plants(NPPs). In order to understand the nature of task complexity, task complexity factors need to be identified and organized in a systematic manner. Although several methods have been suggested for identifying and organizing task complexity factors, it is rare to find an analytical approach based on a theoretically sound model. Method: This study regarded a task as a system to be designed. Three levels of design abstraction, which are functional, behavioral, and structural level of a task, characterize the design aspects of a task. The behavioral aspect is further classified into five cognitive processing activity types(information collection, information analysis, decision and action selection, action implementation, and action feedback). The complexity dimensions describe a task complexity from different perspectives that are size, variety, and order/organization. Combining the design aspects and complexity dimensions of a task, we developed a model from which meaningful task complexity factors can be identified and organized in an analytic way. Results: A model consisting of two facets, each of which is respectively concerned with design aspects and complexity dimensions, were proposed. Additionally, twenty-one task complexity factors were identified and organized based on the model. Conclusion: The model and approach introduced in this paper can be effectively used for examining human performance and humansystem interface design issues in NPPs. Application: The model and approach introduced in this paper could be used for several human factors problems, including task allocation and design of information aiding, in NPPs and extended to other types of complex systems such as air traffic control systems as well.
\end{abstract}

Keywords: Task complexity, Complexity factors, Complexity model, Human performance

\section{Introduction}

원자력발전소(NPPs: Nuclear Power Plants)와 같은 대 형시스템의 안전성과 생산성을 확보하는데 인적 수행도의
향상은 필수적인 요소로 인식되고 있다(Ham et al., 2011). 따라서 인적 수행도를 분석하고 예측하는 것은 많은 연구자 들의 관심의 대상이었다(Wickens, 1992). 다양한 요소들이 인적 수행도에 직간접적으로 영향을 미치고 있음은 많은 연 구들을 통해 알려져 있다(Sheridan, 2002; Vicente, 1999).

Corresponding Author: Dong-Han Ham. Department of Industrial Engineering, Chonnam National University, Gwangju, $500-757$.

Mobile: +82-16-417-4607, E-mail: donghan.ham@gmail.com

Copyright@2013 by Ergonomics Society of Korea(pISSN:1229-1684 eISSN:2093-8462). All right reserved.

(c) This is an open-access article distributed under the terms of the Creative Commons Attribution Non-Commercial License(http://creativecommons.org/licenses/by-nc/3.0/), which permits unrestricted non-commercial use, distribution, and reproduction in any medium, provided the original work is properly cited. http://www.esk.or.kr 
그 중에서도 복잡도는 인적 수행도에 다양한 방식으로 영향 을 미치는 대표적인 요소이다(Park, 2009). 그러므로 대형 시스템에서의 인적 수행도의 향상을 위해서는 복잡도에 관 한 체계적인 연구가 필수적이라 할 수 있다.

대형시스템에서 복잡도를 정확하게 정의한다는 것은 결코 쉬운 일이 아니다. 그래서 많은 복잡도 연구들은 복잡도에 대한 다양한 정의 하에서 연구가 진행되어 왔다(Hollnagel and Woods, 2005). 그러한 다양한 정의들의 차이는 그 정 의들이 복잡한 인간-시스템 상호작용의 어떤 측면을 중점 적으로 다루고 있는가에서 비롯된다. 예로 Gill and Hicks (2006)는 인지적 인간-시스템 상호작용과 관련해 13개의 복잡도 정의가 있음을 보고했다. 이들간의 차이는 시스템에 서 이루어지는 상호작용 직무의 어떤 측면을 강조하는가에 의해 설명된다.

복잡도 연구와 관련해서 실용적인 목적으로 여러 유형의 복잡도가 분류되어 왔다. 상황인식(Situation Awareness) 으로 유명한 Endsley와 그의 동료들은 기술적인 시스템 복 잡도에서 인간이 실제적으로 인식하는 복잡도에 이르기까지 의 다양한 복잡도를 분류하는 좋은 프레임워크를 제시했다 (Endsley et al., 2003). 그들이 분류한 복잡도의 유형은 다음과 같다: 기술적인 시스템의 기능적 복잡성에 기인하는 기술적 시스템 복잡도(technical system complexity), 운전 원(human operators)이 시스템을 사용하기 위해 다루어야 하는 운영 복잡도(operational complexity), 사용자 인터페 이스에 의해 영향 받으면서 운전원이 직접적으로 느끼게 되 는 명백한 복잡도(apparent complexity). 명백한 복잡도 는 인지적 복잡도(cognitive complexity), 디스플레이/인 식 복잡도(display/perceptual complexity), 반응 복잡도 (reaction complexity)의 세 형태로 더 구체화된다. Li and Wieringa(2000)의 연구도 비슷한 방식으로 여러 복잡도 의 유형을 구분하고 있다. 그들이 구분한 복잡도의 유형은 다음과 같다: 직무복잡도(task complexity), 공정 복잡도 (process complexity), 제어시스템 복잡도(control system complexity), 인터페이스 복잡도(human-machine interface complexity), 주관적으로 인식된 복잡도(perceived complexity).

위에서 언급한대로 여러 유형의 복잡도가 분류되어 왔지 만 객관적 복잡도(objective complexity)와 주관적 복잡 도(subjective complexity) 의 구분이 인적 수행도 연구와 관련해 가장 의미 있는 구분 중의 하나일 것이다 $(\mathrm{Li}$ and Wieringa, 2000). 객관적 복잡도는 기술적으로 설계된 특 징과 상황적 요소에 의해서만 형성이 되는 복잡도이다. 반면 에 주관적 복잡도는 주어진 객관적 복잡도를 어느 정도로 복 잡하게 실제적으로 느끼고 인식하는가에 대한 개념이다. 따 라서 주관적 복잡도는 인간의 지식과 경험에 크게 의존할
수 있다. 동일한 정도의 객관적 복잡도가 여러 사람들에게 다른 정도의 주관적 복잡도로 인식될 수 있다는 사실은 인 적 수행도의 평가 및 예측에서 매우 중요한 의미를 지닌다 (Rasmussen and Lind, 1981).

그런데 객관적 복잡도와 주관적 복잡도를 종합적으로 고 려할 수 있다면 인적 수행도와 복잡도의 관련성 연구에서 매우 유익할 것이다. 직무복잡도(task complexity)의 개념 이 이러한 목적으로 유용하게 활용될 수 있을 것이다(Ham et al., 2011a, 2011b; Liu and Li, 2012; Park, 2009; Park and Jung, 2007). 운전원이 상호작용하는 시스템의 기술적 특징으로부터 주로 기인하는 객관적 복잡도는 시스템 내에 서 운전원이 수행해야 하는 직무를 통해 그 복잡도의 특성 이 많이 반영된다. 원자력발전소에서의 경우를 예로 들면 절 차적 직무(procedure-based tasks) 뿐만 아니라 원리적 지 식기반의 직무(principle knowledge-based tasks)의 모두 에서 발전소시스템의 객관적 복잡도의 특성이 많이 반영되 어 있음을 알 수 있다. 또한 그 직무를 수행하는 과정에서 운전원이 실제적으로 객관적 복잡도의 특성을 느끼고 인지 하면서 주관적 복잡도를 형성한다고 볼 수 있다. 그러므로 직무복잡도는 객관적 복잡도와 주관적 복잡도의 의미적 차 이 (semantic gap)를 연결해주는 유용한 연결고리의 역할을 한다고 할 수 있다.

앞의 설명으로부터 대형시스템에서의 인간-시스템 상호 작용과 관련된 다양한 복잡도 중에서 직무복잡도의 연구가 중요한 의미를 지닌다는 것을 알 수 있다. 그런데 현재 대형 시스템에서 발생하는 인간-시스템 상호작용에서의 인간의 직무는 대부분 지식 및 정보를 처리하는 인지적 직무의 특징 을 지닌다. 따라서 직무복잡도는 인간의 인지적 정보처리의 관점에서 해석될 필요성이 있다. 또한 직무복잡도는 사용자 인터페이스 및 정보지원시스템 등에 의해 그 정도가 조정될 수 있다. 어떤 직무에서 처리해야 하는 정보요건을 사용자 인터페이스나 정보지원시스템에서 적절하게 제공해준다면 해당 직무의 복잡도는 상당히 감소할 가능성이 있다(Xing, 2004).

다른 유형의 복잡도와 마찬가지로 직무복잡도도 다양한 요소에 의해 영향을 받는다. 직무복잡도의 특성을 보다 잘 이해하고 체계적으로 평가하기 위해서 직무복잡도 요소를 파악하고 이들의 관련성을 이해하는 것은 필수적인 일이다. 이러한 문제의식을 갖고 많은 연구들이 직무복잡도 요소를 파악하는데 기여해 왔다. 예로 Campbell(1988)은 인지적 직무를 복잡하게 만드는 4 가지의 요소를 파악했다: 직무의 목표상태로 가기 위한 다양한 경로의 수, 직무의 다양한 목 표상태의 수, 상호 연관되어 충돌하는 경로들, 경로와 목표 상태의 관계에서의 불확실성. 그는 이러한 4 가지의 복잡도 요소를 바탕으로 16 개의 복잡한 인지적 직무를 구분했다. 
특정 상황 혹은 조건에서의 직무를 복잡하게 만드는 요소 들도 여러 연구에 의해 파악되었다(Liu and Li, 2012). 예로 Hillburn (2004)은 항공관제시스템에서의 직무복잡도 요소를 광범위하게 파악하고 분류했다. Park(2009)은 원자력발전 소와 같은 공정제어시스템에서의 직무복잡도 요소를 5 가지 로 분류하고 그에 기반해서 TACOM(TAcom COMplexity) 이라는 복잡도 정량화 척도를 개발하였다. Thelwell(1994) 의 연구는 공정제어시스템의 직무복잡도에 영향을 미치는 요소에 대한 좋은 연구의 또 다른 예이다. 현재까지 대부분 의 연구가 개인적인 직무 상황에서의 복잡도에 영향을 미치 는 요소를 연구해 왔다. 그러나 최근에 팀에 기반한 직무의 중요성이 중요해지면서 팀 직무복잡도에 영향을 미치는 요 소들에 대한 연구도 증가하고 있다. 예로 Rothrock et al. (2005)의 연구는 팀 직무복잡도에 영향을 미치는 11 개의 요소를 파악하고 이들을 세 개의 그룹(직무의 범위, 직무의 구조화, 직무의 불확실성)으로 조직화하였다.

현재까지 많은 연구들의 직무복잡도 요소를 파악해 왔으 며 이들은 분명히 직무복잡도의 특성을 파악하고 직무복잡 도를 낮추는 설계활동에 상당한 기여를 해왔다. 그러나 연구 방법론적인 측면에서 이들은 대부분 경험적인 방법에 의해 직무복잡도 요소를 파악해왔다. 그래서 해당 직무복잡도 요 소가 도출된 특정 상황에서는 그 요소가 의미가 있지만 다 른 상황에서는 그렇지 않을 가능성이 높다. 즉 외부타당성 (external validity)이 낮을 수 밖에 없다. 또한 그러한 요소 들이 어떤 관련성을 갖고 있는가를 파악하는 것도 쉽지 않다. 따라서 여러 연구자들이 직무복잡도의 요소를 분석적으로 파악하고 조직화하는데 활용할 수 있는 직무복잡도 모형을 개발할 필요성이 있음을 강조했다(Campbell, 1994; Ham et al., 2012; Horsky et al., 2003; Liu and Li, 2012).

이러한 문제의식을 갖고 Ham et al.(2012)은 대형시스템 에서의 직무복잡도 요소를 파악하고 조직화하는데 유용하게 활용할 수 있는 직무복잡도 모형을 개발했다. 또한 이 모형 에 기반해서 직무복잡도 요소를 파악하는데 도움이 되는 프 로세스를 제안하였고 21 개의 의미 있는 직무복잡도 요소를 도출하였다. 이 연구에서 개발된 모형과 모형기반의 직무복 잡도 요소 파악 및 구조화 방법은 기존의 연구에 비해 여러 고유한 특성을 지니고 있다. 본 논문에서는 Ham et al. (2012)의 연구에서 제안된 직무복잡도 모형과 그 모형에 기반한 직무복잡도 요소의 파악 및 구조화 방법을 소개한다. 동시에 그 모형과 방법을 보다 구체화하고 그 것들의 유용성 을 높이기 위한 방법을 제안한다.

\section{Model-Based Approach}

직무복잡도 요소의 파악 및 구조화를 위한 직무복잡도 모 형을 개발하기 위해 고려되어야 하는 점이 있다. 첫째로 직 무복잡도라는 것 자체가 추상적인 개념이면서 창발적 속성 (emergent property)을 지니는 것이다. 그래서 직무복잡도 를 구성하는 요소를 완벽하게 파악하기도 어렵고 그것들을 단순하게 합산함으로써 직무복잡도를 측정하는 것이 큰 의 미가 없을 수 있다. 그럼에도 불구하고 직무복잡도를 측정하 는데 있어 직무복잡도 요소를 개별적으로 파악하는 것(일종 의 환원적 접근(reductionism approach))은 필수불가결한 작업이다. 이러한 접근의 단점은 직무복잡도 요소들간의 관 련성을 일관성 있게 파악하기 쉽지 않다는 것이다. 또한 직 무 그 자체와 파악된 요소들간의 의미 있는 관계를 파악하 는 것도 쉽지는 않다. 이러한 단점을 보완하기 위한 방법은 일종의 전체적 접근(holistic approach)을 적용하는 것이다 (Waring, 1996). 즉 직무복잡도 요소들간의 관계를 일관성 있게 제공해 주고 그 요소들이 어떻게 직무복잡도에 영향을 미치는가에 대한 모형을 활용하는 것이다. 이러한 의미에서 직무복잡도 모형은 시스템 사고에 기반해 개발될 필요가 있 다. 둘째로 직무복잡도와 그 요소들을 연구하는 목적은 다양 하지만 궁극적인 목적은 직무를 효과적으로 설계해서 가급 적 직무복잡도를 낮추고 직무의 정보 및 지식요건과 연계해 서 인터페이스 및 정보지원에 활용함이다. 그래서 직무복잡 도 모형과 그 모형으로부터 파악된 요소들은 직무의 설계 과 정에 유용하게 활용될 필요가 있다. 셋째로 직무복잡도를 여 러 관점에서 바라볼 필요가 있다는 것이다. 직무복잡도가 한 관점에서 모든 것을 설명할 수 있는 개념은 아니라는 것이다.

본 논문에서 소개하는 직무복잡도 모형은 위에서 언급한 요건에 기반해 개발되었다(Ham et al., 2012). 이 모형은 환원적 접근법과 전체적 접근법이 동시에 고려될 수 있는 여 건을 제공한다. 직무를 분석하고 그 구성 요소를 파악한 후 에 이로부터 직무복잡도 요소를 도출하는 것이 가장 실용 적인 환원적인 접근법의 복잡도 요소 파악일 것이다. 특히 현대의 인간-시스템 상호작용의 직무는 대부분 인지적인 직무이므로 인지적 직무분석을 수행할 필요가 있다. 이러한 의미에서 이 모형은 계층적 직무분석(HTA: Hierarchical Task Analysis) (Shepherd, 1998)과 SGT(Sub-GoalTemplate) 분석 (Omerod and Shepherd, 2004)을 활용 해 직무분석이 이루어져야 함을 권고한다. 이러한 분석결과 로부터 직무복잡도 요소들에 대한 기본정보를 구한 후에 모 형을 활용한 직무복잡도 요소의 파악 및 구조화를 강조한다. 또한 이 모형은 두 개의 축으로 구성된다. 하나는 직무의 설 계 측면(design aspects of a task)이고 다른 하나는 직무의 


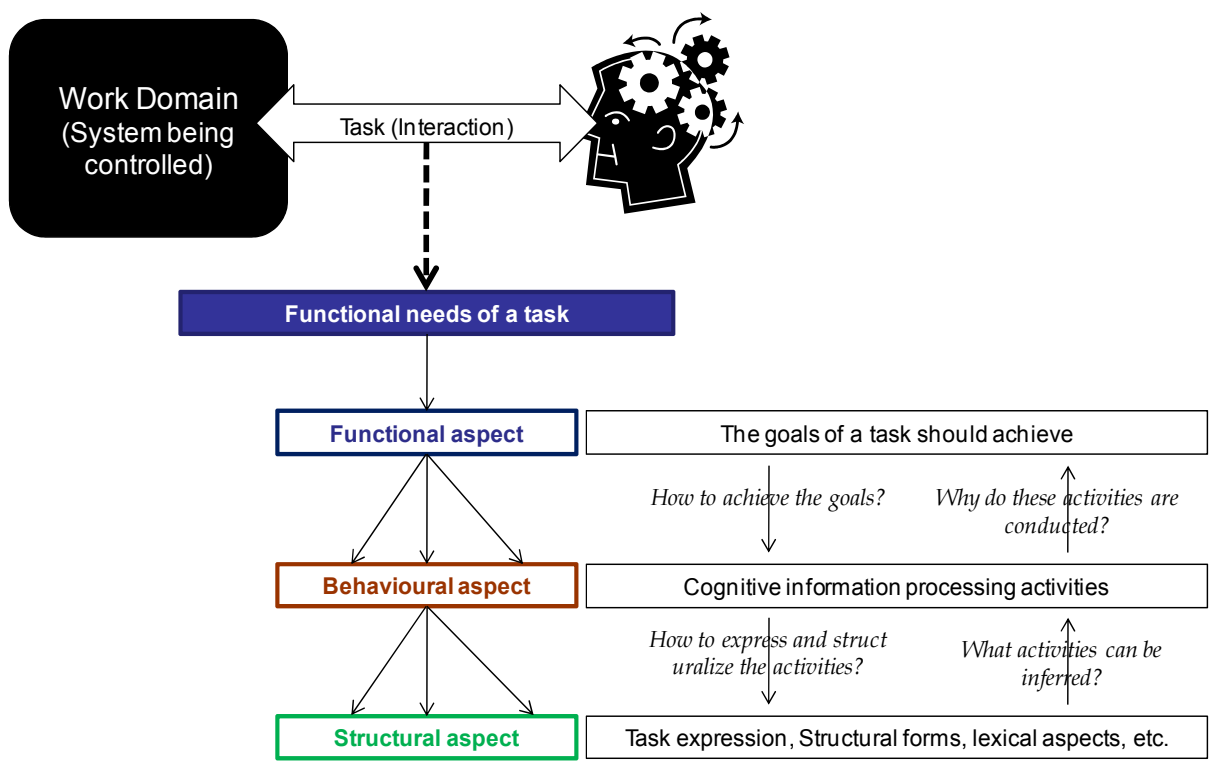

Figure 1. Three design aspects of a task

복잡도 차원 (design dimensions of a task)이다.

본 논문에서 소개하는 직무복잡도 모형의 전반적인 내용 을 위에서 설명했고 다음으로 보다 구체적인 내용을 기술하 고자 한다.

\subsection{Design aspects and complexity dimensions of a task}

직무 그 자체를 설계되는 일종의 시스템으로 고려할 때 시스템공학 혹은 설계과학 분야에서의 설계과정 모형을 직 무 개념에 적용해볼 수 있다. 또한 그 설계과정 모형에서 인지적 직무의 특성이 고려할 수 있어야 한다. 이러한 점을 고려할 때 여러 설계과정 모형 중에서 기능-행태-구조 (FBS: Function-Behavior-Structure) 프레임워크(Gero and Kannengiesser, 2004; Vermaas and Dorst, 2007)가 직무복잡도 모형을 위해 유용하게 활용될 수 있다.

FBS 프레임워크는 시스템 혹은 설계물(artifact)의 세 가 지 설계 측면을 구분한다: 기능, 행태, 구조. 시스템의 기능 측면은 해당 시스템이 설계된 근본적인 목표에 관련된다. 행 태 측면은 시스템의 설계 측면으로부터 기대되거나 도출되 는 속성을 의미하며 실제로 시스템이 행동하는 것과 관련된 다. 구조적 측면은 가시적인 시스템의 구성 요소와 그들간의 관련성과 관련된다. 즉 $\mathrm{FBS}$ 프레임워크는 시스템의 설계 측 면을 설계지식의 추상화 관점에서 세 가지 수준으로 구분한 것으로도 해석된다. 또한 세 가지 디자인 측면의 관계는 목 적-수단 관계(goal-means relations) 의 관점에서 해석될 수 있다. 이런 점에서 인지시스템공학에서 시스템지식의 모
형화 기법으로 많이 활용되는 Rasmussen(1985)의 추상화 계층(AH: Abstraction Hierarchy) 와 의미상 매우 유사하다 할 수 있다.

$\mathrm{FBS}$ 프레임워크는 설계 과정은 기능과 행위, 행위와 구조 간의 관계를 설정하는 것으로 해석한다. 이 과정을 더 체계 적으로 설명하기 위해 $\mathrm{FBS}$ 프레임워크는 5 개의 설계 상태 와 8 개의 설계 변환 과정을 구분하다. 5 개의 설계 상태는 기 능 $(\mathrm{F})$, 예측되는 행태 $\left(\mathrm{B}_{\mathrm{e}}\right)$, 구조로부터 도출되는 행태 $\left(\mathrm{B}_{\mathrm{s}}\right)$, 구조 $(\mathrm{S})$, 문서화된 상태(D). 8 개의 변환 과정은 5 개의 설 계 상태들간의 전이 과정으로 해석된다. 그들은 다음과 같 다: 형성 (formulation; $\mathrm{F} \rightarrow \mathrm{B}_{\mathrm{e}}$ ), 통합 (synthesis; $\mathrm{B}_{\mathrm{e}} \rightarrow$ $\mathrm{S})$, 분석 (analysis; $\mathrm{S}->\mathrm{B}_{\mathrm{S}}$ ), 평가(evaluation; $\mathrm{B}_{\mathrm{e}}\langle->$ $\mathrm{B}_{\mathrm{S}}$ ), 서류화(documentation; $\mathrm{S}->\mathrm{D}$ ), 제 1 유형의 재형 성 (reformulation type $1 ; \mathrm{S}->\mathrm{S}$ ), 제 2 유형의 재형성 (reformulation type 2; $\mathrm{S} \rightarrow \mathrm{B}_{\mathrm{e}}$ ), 제 3 유형의 재형성 (reformulation type 3; $\mathrm{S}->\mathrm{F}$ ).

$\mathrm{FBS}$ 프레임워크를 직무설계에 응용하면 직무도 세 가지 의 설계 측면으로 해석이 가능하다. 또한 직무가 설계되는 과정도 5 개의 설계 상태와 8 개의 변환 과정으로 설명할 수 있다. 직무복잡도 요소들을 이러한 설계 측면, 설계 상태 및 변화 과정의 관점에서 해석이 가능하다면 직무복잡도 요소 들의 측정 및 평가로부터 직무설계에 의미 있는 정보를 구 할 수 있을 것이다. Figure 1은 직무의 세 가지 측면과 그들 간의 관련성을 보여준다. 직무의 기능적 측면은 시스템 내에 서 인간이 수행하는 직무를 통해 달성하려고 하는 목적과 관 련이 있다. 직무의 구조적 측면은 실제로 직무가 설계된 구 


\begin{tabular}{|c|c|c|c|c|c|}
\hline \multirow{2}{*}{\multicolumn{2}{|c|}{ Design Aspect of Task }} & \multicolumn{3}{|c|}{ Complexity Dimension } & \multirow{2}{*}{ Note } \\
\hline & & Size & Variety & Order/Organization & \\
\hline \multicolumn{2}{|c|}{ Functional Aspect of Task } & & & & Related to task level \\
\hline \multirow{5}{*}{$\begin{array}{c}\text { Behavioural } \\
\text { Aspect of Task } \\
\text { (Part of cognitive } \\
\text { activities to be } \\
\text { considered for } \\
\text { Automation or } \\
\text { Aiding) }\end{array}$} & Information Collection & & & & \multirow{5}{*}{$\begin{array}{c}\text { Related to step level } \\
\text { (Selection of complexity } \\
\text { factors is dependent on } \\
\text { the objective of actions } \\
\text { (action verbs)) }\end{array}$} \\
\hline & Information Analysis & & & & \\
\hline & $\begin{array}{l}\text { Decision and Action } \\
\text { Selection }\end{array}$ & & & & \\
\hline & Action Implementation & & & & \\
\hline & Action Feedback & & & & \\
\hline \multicolumn{2}{|c|}{ Structural Aspect of Task } & & & & Related to task level \\
\hline
\end{tabular}

Figure 2. Model for identifying and organizing task complexity factors

조(예로 직무의 절차가 기술된 절차서의 형식)가 될 것이다. 직무의 행태적 측면은 직무의 목적을 달성하기 위해 인간이 실제로 수행해야 하는 구체적인 내용이 될 것이다. 대부분의 직무의 목표는 정보 및 지식의 처리일 것이므로 구체적인 인 지적인 직무들(예: 정보의 수집, 의사결정)이 행태적 측면을 구성하게 된다.

한편 복잡도는 여러 관점에서 해석될 필요가 있다. 어떤 관점에서 복잡도를 해석하는 것이 올바른가에 대한 문제 도 명확한 해답은 없다. 그러나 많은 연구들이 복잡도는 세 가지 차원에서 고려될 필요가 있음을 강조한다: 크기 (size), 다양성 (variety), 조직화정도(order/organization) (Zamenopoulos and Alexiou, 2005). 시스템을 구성하는 요소가 많을수록 대체적으로 복잡도는 증가하는 경향을 지 닌다. 그 구성 요소들이 다양할수록 또한 복잡도는 증가할 것이다. 또한 그 구성 요소들간의 관련성이 잘 조직화되어 있고 정련화되어 있다면 복잡도는 낮아질 것이다. 이런 관점 에서 직무복잡도도 세 가지 차원에서 고려될 필요가 있다: 직무를 구성하는 세부단위의 요소들 혹은 직무가 처리해야 하는 정보의 수, 그 요소들 혹은 정보들의 다양성, 그들간의 관련성이 갖고 있는 조직화 정도.

\subsection{Model of complexity factors}

앞에서 논의된 직무복잡도 모형의 요건과 모형을 구성하 게 될 두 가지 측면의 이론적 기반에 근거해서 Figure 2에 서 보여지는 직무복잡도 모형이 개발되었다(Ham et al., 2012).

이 모형에서는 직무의 기능적, 행태적, 구조적 측면을 구
분함과 동시에 직무복잡도를 세 가지 차원에서 고려할 수 있도록 해준다. 직무의 설계 측면으로부터 각 측면과 관련된 직무복잡도 요소가 직무복잡도에 어떻게 영향을 미치는가를 파악하는가에 대한 정보를 얻을 수 있다. 또한 직무설계의 어떤 측면을 개선해야 전체적인 직무복잡도를 감소시킬 수 있는가에 대한 정보도 얻을 수 있다. 직무의 설계 측면과 직 무복잡도 차원이라는 두 개의 축으로 구성된 이 모형은 직무 복잡도 요소들간의 관련성을 전체 직무복잡도와 인적 수행 도의 관점에서 일관성 있게 종합적으로 해석할 수 있는 여 건을 제공한다. 그런데 이 모형과 관련해 두 가지 점이 고려 될 필요가 있다. 첫째는 직무의 행태적 측면과 관련된 것이 다. 이 모형에서는 직무의 행태적 측면을 인지적인 정보처리 활동과 관련해서 5 가지의 세부 활동으로 구분한다: 정보수 집 (information collection), 정보분석 (information analysis), 의사결정 및 행동선택(decision and action selection), 행 위수행(action implementation), 행위피드백 관찰(action feedback). 이 5 가지 인지적 정보처리 활동의 구분은 Rasmussen의 의사결정나무(decision ladder) (Rasmussen et al., 1994)를 본 연구의 목적에 맞게 단순화한 것이다.

두 번째로 고려되어야 하는 것은 직무의 구조와 직무의 설계 측면과의 관련성이다. Figure 3 은 원자력발전소에서의 증기발생기 튜브파손(SGTR: Steam Generation Tube Rupture)에 대응하기 위한 직무절차서의 단면을 보여준다. 이 직무절차서에 나와 있듯이 대형시스템의 직무의 구조는 세 단계로 구분된다. 하나의 직무는 여러 세부 직무로 구성 되는데 이러한 세부 직무를 스텝(step)이라 한다. 또 하나의 스텝은 다시 여러 세부 스텝으로 구성되는데 이러한 세부 스 텝을 액션(action)이라 한다. 보통 하나의 액션은 액션을 특 


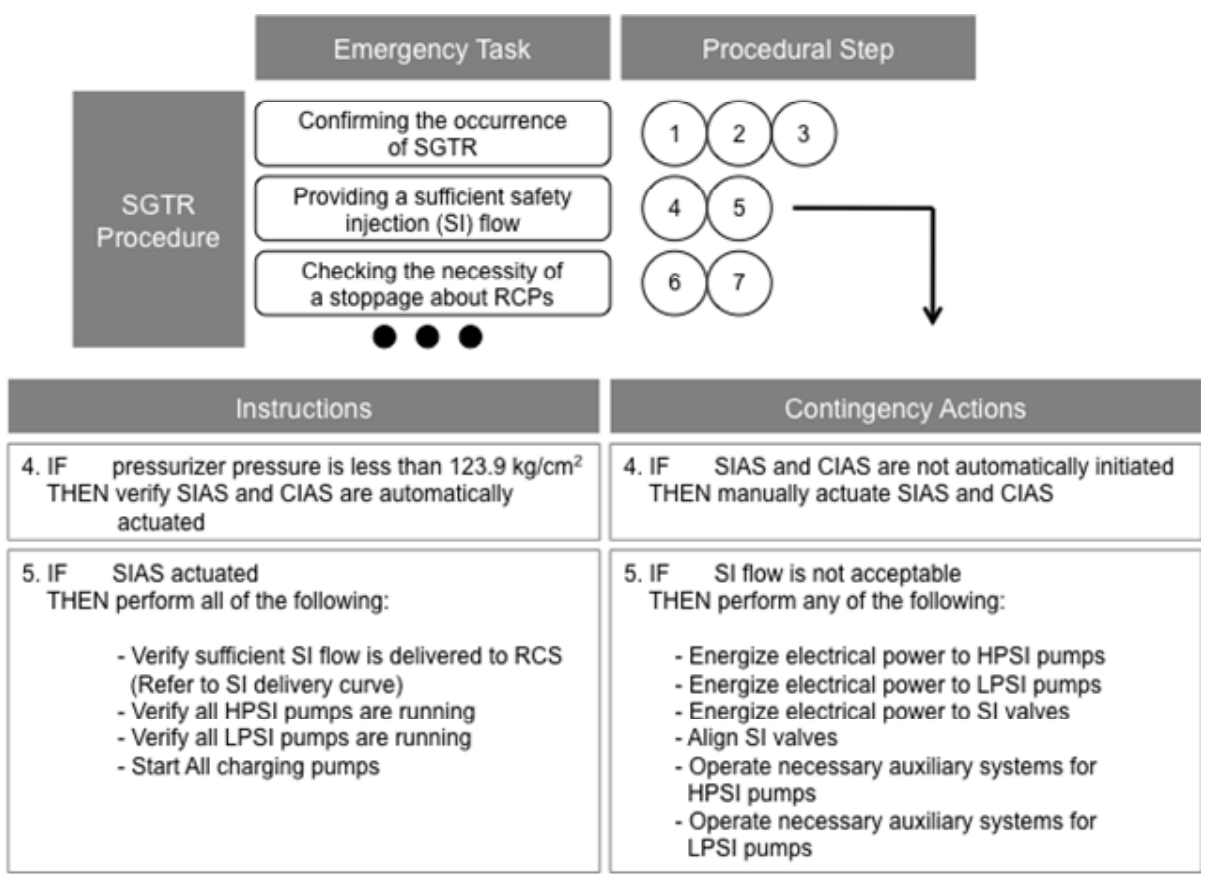

SIAS: Safety Injection Actuation Signal; CIAS: Containment Isolation Actuation Signal HPSI: High Pressure Safety Injection; LPSI: Low Pressure Safety Injection

Figure 3. Part of task procedure for coping with a Steam Generation Tube Rupture(SGTR)

성화하는 동사와 그 동사의 대상으로 구성된다(예: 펌프 $\mathrm{A}$ (대상)를 구동시켜라(동사)). 이러한 세 단계의 직무 구조를 고려했을 때 직무의 기능적 측면과 구조적 측면과 관련된 직무복잡도 요소는 직무의 직무 수준(task level)의 단계에 서 파악되면 충분하다. 그러나 직무 수준의 단계는 해당 직 무의 목적을 달성하기 위한 세부적인 인지적인 정보처리 활 동과 관련된 직무복잡도 요소를 파악하기에는 그 수준이 너 무 크다. 그래서 직무의 행태적 측면과 관련된 직무복잡도 요소는 스텝 수준(step level)에서 고려될 필요가 있다. 그런 데 하나의 스텝에서 5 개의 인지적 활동이 모두 수행되는 것 은 아니므로 직무분석을 통해 해당 스텝과 관련 있는 인지활 동을 선별하는 것이 필요하다.

두 가지 축으로 구성된 모형에서는 총 21 개의 직무복잡도 요소가 파악될 필요가 있음을 규정한다. 각 복잡도 요소는 전체적인 직무복잡도에 고유한 의미를 지닌다. 그런데 각 직 무복잡도 요소를 파악하기 위해서는 앞에서 설명된 것처럼 직무분석(인지적 직무분석)이 먼저 선행되어야 한다. 직무분 석에서 얻어진 정보를 바탕으로 고유한 21 개의 직무복잡도 요소가 파악된다는 것은 환원적 접근법을 활용한다는 것이 다. 또한 이렇게 파악된 요소들은 모형의 두 가지 축에서 제 공하는 개념들을 이용해 유기적으로 연결되고 전체 직무복 잡도와의 관련성이 해석될 수 있다. 이런 의미에서 개발된
모형은 전체적 접근법의 활용을 지원한다고 할 수 있다.

\subsection{Complexity factors based on a model}

앞에서 소개된 직무복잡도 모형은 직무복잡도 요소를 보 다 체계적으로 분석적인 방법으로 파악하고 구조화하는데 필요한 일종의 개념적 요건을 명시하는데 주 목적이 있다.

각각 고유한 의미를 지니는 총 21 개의 직무복잡도 요소가 도출되어야 함은 명시하지만 그 복잡도 요소가 무엇이어야 한다는 것은 명시하지 않는다. 이러한 요소를 도출하기 위해 서는 직무분석의 정보도 활용해야 하고 분석가의 경험 및 주 관적 판단 등이 모두 요구된다고 할 수 있다. 그러나 개발된 모형을 활용하는데 도움을 주고자 Ham et al. (2012)은 직무 복잡도의 기존의 연구결과와 원자력발전소에서의 복잡도 연 구 경험을 바탕으로 21 개의 복잡도 요소를 Figure 4 와 같이 제안하였다. 이 복잡도 요소들은 하나의 예이고 직무복잡도 요소 파악의 목적과 분석가의 관점에 따라 다른 요소들이 충 분히 도출될 수 있다. 그러나 도출된 요소들이 다르다고 해 도 각 요소들의 의미와 그들간의 관련성은 모형에서 규정한 원칙과 개념에 부합되어야 한다

제안된 21 개의 직무복잡도 요소에 대한 자세한 설명은 참 고문헌 $\operatorname{Ham}$ (2010)에서 구할 수 있다. 제안된 복잡도 요소 


\begin{tabular}{|c|c|c|c|c|}
\hline \multirow{2}{*}{\multicolumn{2}{|c|}{ Design Aspect of Task }} & \multicolumn{3}{|c|}{ Complexity Dimension } \\
\hline & & Size & Variety & Ordrder/Organization \\
\hline \multicolumn{2}{|c|}{ Functional Aspect of Task } & $\begin{array}{l}\text { Span of Abstraction- } \\
\text { Decomposition space }\end{array}$ & Number of task goals & Number of precoditions \\
\hline \multirow{5}{*}{$\begin{array}{c}\text { Behavioural Aspect of } \\
\text { Task } \\
\text { (Part of cognitive } \\
\text { activities to be } \\
\text { considered for } \\
\text { Automation or } \\
\text { Aiding) }\end{array}$} & Information Collection & $\begin{array}{l}\text { Number of variables to } \\
\text { collect/identify }\end{array}$ & $\begin{array}{c}\text { Number of different types of } \\
\text { collected variables }\end{array}$ & $\begin{array}{l}\text { Number of sources to collect } \\
\text { information }\end{array}$ \\
\hline & Information Analysis & $\begin{array}{l}\text { Number of variables to be } \\
\text { derived/produced cognitively }\end{array}$ & $\begin{array}{c}\text { Number of different types of } \\
\text { derived variables }\end{array}$ & $\begin{array}{l}\text { Number of cognitive } \\
\text { operations to be conducted }\end{array}$ \\
\hline & $\begin{array}{l}\text { Decision and Action } \\
\text { Selection }\end{array}$ & $\begin{array}{l}\text { Number of decision making } \\
\text { variables }\end{array}$ & $\begin{array}{c}\text { Number of possible paths to } \\
\text { achieve a step goal }\end{array}$ & $\begin{array}{c}\text { Number of subjective } \\
\text { judgment needed for decisions }\end{array}$ \\
\hline & Action Implementation & $\begin{array}{c}\text { Number of control items to act } \\
\text { on }\end{array}$ & $\begin{array}{l}\text { Number of different types of } \\
\text { control titems }\end{array}$ & $\begin{array}{c}\text { Number of considerations to } \\
\text { be paid at the same time }\end{array}$ \\
\hline & Action Feedback & $\begin{array}{l}\text { Number of variables to } \\
\text { confirm }\end{array}$ & $\begin{array}{l}\text { Number of different types of } \\
\text { variables to be confirmed }\end{array}$ & $\begin{array}{c}\text { Number of sources to get } \\
\text { feedback from }\end{array}$ \\
\hline \multicolumn{2}{|c|}{ Structural Aspect of Task } & Number of steps & Logical relation between steps & $\begin{array}{l}\text { Number of warning or } \\
\text { referential information }\end{array}$ \\
\hline
\end{tabular}

Figure 4. Twenty-one task complexity factors identified from the model

\begin{tabular}{|c|c|c|c|c|c|c|}
\hline \multirow{2}{*}{\multicolumn{2}{|c|}{$\begin{array}{r}\text { Design Aspect } \\
\text { Complexity Dimensions }\end{array}$}} & \multicolumn{5}{|l|}{ Functional Aspect of Task } \\
\hline & & \multicolumn{5}{|l|}{ Variety } \\
\hline Factor Name & Purpose of Factor & Method of Application & Measurement and Formula & $\begin{array}{l}\text { Interpretation of } \\
\text { Measured Value }\end{array}$ & $\begin{array}{c}\text { Metric } \\
\text { Scale Type }\end{array}$ & $\begin{array}{c}\text { Measure } \\
\text { Type }\end{array}$ \\
\hline $\begin{array}{c}\text { Number of task } \\
\text { goals }\end{array}$ & $\begin{array}{c}\text { How many goals } \\
\text { does this task } \\
\text { achieve? } \\
\text { How many goals are } \\
\text { related to this single } \\
\text { task? }\end{array}$ & $\begin{array}{l}\text { Examine the part of } \\
\text { 'Objective' in the } \\
\text { procedures for the task; } \\
\text { Count the task goals or } \\
\text { situations for which the } \\
\text { procedure should be used }\end{array}$ & $\begin{array}{l}X=\text { the number of task goals } \\
\text { that should be achieved by } \\
\text { conducting the task }\end{array}$ & $\begin{array}{l}\quad X>=1 \\
\text { The larger is the } \\
\text { more complex; } \\
\text { The most desirable is } \\
X=1\end{array}$ & Absolute & $X=$ Count \\
\hline \multicolumn{7}{|c|}{$\begin{array}{l}\text { Note: } \\
\text { 1. It is usual that several task goals are described in one sentence and not explicitly specified in the 'Objective' part in the procedure; thus it should be } \\
\text { carefully examined through reading procedures. }\end{array}$} \\
\hline
\end{tabular}

Figure 5. Example of detailed description and guidance of a task complexity factor

의 의미를 구체화하고 실제로 사용하는데 도움을 주고자 또 한 각 요소 사용에 대한 일종의 지침을 Figure 5 와 같이 마 련했다. Figure 5는 직무의 기능적 측면과 다양성의 복잡도 차원과 연관되어 있는 복잡도 요소 '직무 목표의 수(number of task goals)'에 대한 지침의 예이다.

\section{Process of Using the Model and Task Complexity Factors}

소개된 직무복잡도 모형을 활용해 직무복잡도 요소를 파
악하고 구조화하는 방법과 그 요소들을 측정하고 그 결과를 활용하는 방법은 다양할 수 있다. 그러나 이 모형을 활용하 는 과정의 표준적인 방법을 Figure 6 과 같이 요약할 수 있 다. 이 방법은 제안하는 표준적인 방법이므로 반드시 이 과 정을 따를 필요는 없다.

첫째로 인지적 직무분석이 체계적으로 상세하게 이루어져 야 한다. 직무분석의 결과는 직무복잡도 요소 파악 및 구조 화의 기본정보로 활용된다. 이 단계에서 이용 가능한 직무분 석 혹은 인지적 직무분석 기법이 모두 활용될 수 있다. 그러 나 앞에서 언급한 것처럼 $\mathrm{HTA}$ 와 SGT를 활용한 계층적인 직무 구조와 기본적인 인지적 정보요건 도출을 본 연구에서 는 권고한다. 또한 직무의 기능적 측면과 관련한 직무복잡도 


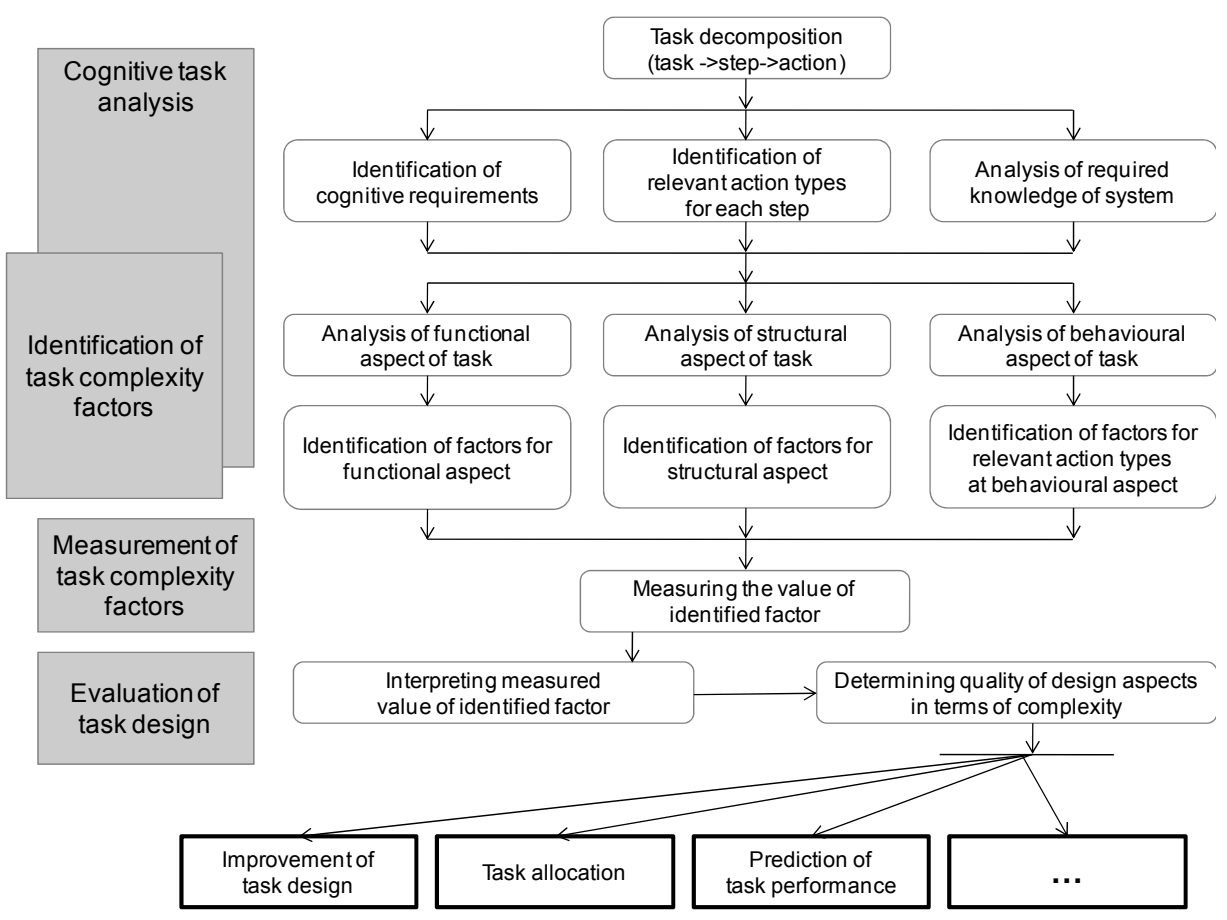

Figure 6. Process of using the model and task complexity factors

요소 도출에서 인간이 상호작용하는 시스템의 지식을 추상 화 계층의 관점에서 분석할 필요가 있다. 그러한 이유로 인 지적 작업분석(CWA: Cognitive Work Analysis)의 작업영 역분석(WDA: Work Domain Analysis)의 수행을 또한 권 고한다.

소개된 직무복잡도 모형은 직무의 설계 측면을 강조하므 로 직무분석 과정에서도 이 점을 고려할 필요가 있다. 직무 의 기능적 측면과 관련해 개별 직무의 목표를 분명하게 파 악할 필요가 있다. 직무의 행태적 측면과 관련해서 해당 직 무를 위한 스텝 및 액션에서 어떤 인지적 정보 및 지식요건 이 필요한가에 대한 구체적인 분석이 이루어져야 한다. 직무 의 구조적 측면은 직무의 구조를 기술하는 다이어그램이나 직무절차서로부터 쉽게 파악될 수 있다. 또한 직무절차에 명 확하게 드러나지 않는 직무 스텝들간의 논리적인 관련성이 있을 수 있으므로 이 점을 주의해야 한다.

둘째로 개발된 모형의 기본 개념을 염두에 두고 복잡도 요 소를 파악하고 조직화한다. 직무의 기능적 측면과 구조적 측 면에 관련된 복잡도 요소는 모두 파악되어야 한다. 그러나 직무의 행태적 측면은 다를 수 있다. 직무를 구성하는 하나 의 스텝을 수행하는데 5 개의 모든 인지적 정보처리 활동이 필요한 것이 아니므로 어떤 활동이 관련 있는가를 먼저 파악 한 후에 해당 활동에 대응되는 복잡도 요소를 파악할 필요가 있다.
셋째로 파악된 복잡도 요소들은 적절한 방식으로 측정이 되어야 한다. $\operatorname{Ham}(2010)$ 의 연구에서 제공된 각 요소의 사 용을 위한 지침의 내용에 근거해 측정할 수 있을 것이다. 그 러나 각 요소를 종합해서 하나의 직무의 복잡도를 정량화하 는 방법은 아직 제안되지 않았다.

넷째로 측정된 복잡도 요소의 값과 그 요소들의 의미 및 그들간의 관련성을 고려해서 직무설계, 인터페이스 설계 및 정보지원 설계 등에 유용하게 활용할 수 있다.

참고문헌 Ham(2010) 과 Ham et al.(2012)는 제안된 모 형 및 21개의 복잡도 요소가 실제로 어떻게 사용될 수 있는 가에 대한 예를 제공하고 있다. Figure 3 에서 소개된 증기발 생기 튜브파손(SGTR)의 직무를 대상으로 직무분석의 결과, 그 결과를 바탕으로 한 직무복잡도 요소의 파악, 파악된 요 소들의 측정 값이 소개되고 있다.

\section{Conclusion}

본 논문에서는 원자력발전소와 같은 대형시스템에서의 직 무복잡도 요소를 도출하고 조직화하는데 활용할 수 있는 직 무복잡도 모형을 소개하였다. 또한 이 모형에 기반해 직무복 잡도 요소를 도출하고 이 요소들간의 관련성을 일관성 있게 
종합적으로 파악할 수 있는 방법을 소개하였다. 소개된 직무 복잡도 모형은 직무의 설계 측면과 직무의 복잡도 차원이라 는 두 개의 축으로 구성되어 있다. 이 두 개의 축으로부터 총 21 개의 고유한 직무복잡도 요소가 도출되어야 함을 규정 한다. 도출된 직무복잡도 요소와 모형에 기반한 그들간의 관 련성은 직무설계, 인터페이스 및 정보지원시스템 설계, 인적 수행도의 분석 등에 유용한 정보를 제공한다.

본 논문에서 소개한 직무복잡도 모형에 기반한 직무복잡 도 요소의 파악 및 구조화 방법은 기존의 경험적인 접근법 에 기반한 방법에 비해 여러 강점을 지닌다. 그러나 그 모형 과 방법의 실용성을 높이기 위해 추가적으로 다음의 연구들 이 필요하다. 첫째로 소개한 직무복잡도 모형에 기반해 직무 복잡도 요소를 파악하고 구조화하는 다양한 실제 사례들을 많이 개발할 필요가 있다. 둘째로 도출된 직무복잡도 요소들 을 활용해 직무 혹은 인터페이스 설계와 평가에 활용하는 방 법에 대한 구체적인 지침이 마련되어야 한다. 셋째로 소개된 모형기반의 방법으로부터 도출된 직무복잡도 요소가 인적 수행도의 예측 및 분석에 얼마나 유용하게 활용될 수 있는 가에 대한 검증이 필요하다. 이를 위해 본 논문에서 소개된 21 개의 직무복잡도 요소들이 해당 직무에서의 인적 수행도 를 얼마나 잘 예측하는가를 실제 인적 수행도 자료를 이용해 연구하는 것은 좋은 추후 연구방향이 될 것이다.

\section{Acknowledgements}

This study was financially supported by Chonnam National University, 2012(Grant Code: 2012-0730).

\section{References}

Campbell, D.J., Task complexity: a review and analysis, Academy of Management Review, 13(1), 40-52, 1988.

Endsley, M.R., Bolte, B. and Jones, D.G., Designing for Situation Awareness: An approach to Human-Centered Design, Taylor \& Francis, 2003.

Gero, J.S. and Kannengiesser, U., The situated function-behavior-structure framework, Design Studies, 25(4), 373-391, 2004.

Gill, T.G. and Hicks, R.C., Task complexity and informing science: a synthesis, Informing Science Journal, 9, 1-30, 2006.

Ham, D-H., Development of Model-Based Method for Evaluating Cognitive Task Complexity and Human-Machine Interface Design in Nuclear Power Plants, KAERI/CM-1253/2009, Korea Atomic Energy Research Institute, 2010.
Ham, D-H., Park, J. and Jung, W., Model-based identification and use of task complexity factors of human integrated systems, Reliability Engineering and System Safety, 100, 33-47, 2012.

Ham, D-H., Park, J. and Jung, W., Extension of TACOM to the complexity of tasks designed for abnormal situations in nuclear power plants, Journal of Loss Prevention in the Process Industries, 24(5), 601-611, 2011a.

Ham, D-H., Park, J. and Jung, W., A framework-based approach to identifying and organizing the complexity factors of human-system interaction, IEEE Systems Journal, 5(2), 213-222, 2011b.

Hillburn, B., Cognitive Complexity in Air Traffic Control: A Literature Review, EEC Note No. 04/04, EUROCONTROL Experimental Center, 2004.

Hollnagel, E. and Woods, D., Joint Cognitive Systems: Foundations of Cognitive Systems Engineering, CRC Press, 2005.

Horsky, J., Kaufman, D.R., Oppenheim, M.I. and Patel, V.L., A framework for analyzing the cognitive complexity of computer-assisted clinical ordering, Journal of Biomedical Informatics, 36(1-2), 4-22, 2003.

Li, K. and Wieringa P.P., Understanding perceived complexity in human supervisory control, Cognition, Technology, and Work, 2(2), 75-88, 2000.

Liu, P. and Li, Z., Task complexity: a review and conceptualization framework, International Journal of Industrial Ergonomics, 47(6), 553-568, 2012.

Ormerod, T.C. and Shepherd, A., Using Task Analysis for Information Requirements Specification: The Sub-Goal Template (SGT) Method. In D. Diaper and N. Stanton (Ed). The Handbook of Task Analysis for Human-Computer Interaction, Lawrence Erlbaum, London, 347 $-365,2004$.

Park, J., The Complexity of Proceduralized Tasks, Springer-Verlag, 2009.

Park, J. and Jung, W. A study on the development of a task complexity measure for emergency operating procedures of nuclear power plants, Reliability Engineering and System Safety, 92(8), 1102-1116, 2007.

Rasmussen, J., The role of hierarchical knowledge representation in decision making and system management, IEEE Transactions on Systems, Man, and Cybernetics, 15, 234-243, 1985.

Rasmussen, J. and Lind, M., Coping with Complexity, RISØ-M-2293, RISØ National Research Laboratory, 1981.

Rasmussen, J., Pejtersen, A.M. and Goodstein, L.P., Cognitive Systems Engineering, John Wiley \& Sons, 1994.

Rothrock, L., Harvey, C.M. and Burns, J., A theoretical framework and quantitative architecture to assess team task complexity in dynamic environments, Theoretical Issues in Ergonomics Science, 6(2), 157-171, 2005.

Shepherd, A., HTA as a framework for task analysis, Ergonomics, 41(11), 1537-1552, 1998.

Sheridan, T.B., Human and Automation: System Design and Research Issues, John Wiley, 2002.

Thelwell, P.J., "What Defines Complexity?", Proceedings of Ergonomics Society's 1994 Annual Conference (pp. 89-94), Warwick. UK. 1994.

Vermaas, P.E. and Dorst, K., On the conceptual framework of John Gero's 
FBS-model and the prescriptive aims of design methodology, Design Studies, 28(2), 133-157, 2007.

Vicente, K.J., Cognitive Work Analysis: Towards Safe, Productive, and Healthy Computer-Based Work, Lawrence-Erlbaum, 1999.

Waring, A., Practical Systems Thinking, International Thomson Business Press, 1996.

Wickens, C.D., Engineering Psychology and Human Performance, 2nd ed., HarperCollins Publishers, 1992.

Xing, J., Measure of Information Complexity and the Implications for Automation Design, DOT/FAA/AM-04/17, US Department of Transportation, 2004.

Zamenopoulos, T. and Alexiou, K., Linking Design and Complexity: A Review, Proceedings of the ECCS 2005 Satellite Workshop (pp. 91 -102), Paris France, 2005.

\section{Author listings}

Dong-Han Ham: donghan.ham@gmail.com; dhham@jnu.ac.kr

Highest degree: $\mathrm{PhD}$, Department of Industrial Engineering, KAIST

Position title: Assistant Professor, Department of Industrial Engineering, Chonnam National University

Areas of interest: Cognitive Systems Engineering, Human-Computer Interaction, Visual Analytics, Knowledge Service Engineering, Service Systems Engineering, and System Safety

Date Received : 2013-01-16

Date Revised :2013-01-30

Date Accepted : 2013-01-30 